\title{
Elemental Abundances of Three Giants in Terzan 7
}

\author{
George Wallerstein
}

Guillermo Gonzalez

University of Washington, Astronomy Department, Box 351580

Seattle, WA 98195, USA

Doug Geisler

Universidad de Concepción, Departmento de Fúsica, Casillo 160-C Concepción, Chile

\begin{abstract}
.
Using VLT UVES spectra of resolving power near 23,000 we have analyzed three stars in Terzan 7 , a globular cluster associated with the Sagittarius Galaxy. We find $[\mathrm{Fe} / \mathrm{H}]=-0.61 \pm 0.1,[\mathrm{O} / \mathrm{Fe}]=+0.25,[\alpha / \mathrm{Fe}]$ $=+0.23 \mathrm{dex}$, and an enhancement of $r / s$ elements as compared with the Sun and Arcturus.
\end{abstract}

\section{Introduction}

Terzan 7 is one of several globular clusters associated with the Sagittarius (Sgr) galaxy (the others are M54, Arp 2, and Terzan 8), a system that appears to be in the process of being captured by the Milky Way. The Sgr galaxy itself seems to have a spread in metallicity. The brightest of its globulars, M54, also shows a spread in metallicity (Layden and Sarajedini 2000). Terzan 7 has a relatively red giant branch indicating a relatively high metallicity in contrast to the blue giant branch of Terzan 8 and the wide giant branch of M54.

During the early evening hours of a VLT UVES observing run devoted to another star system, we obtained one spectrum of each of 3 members of Terzan 7. The spectra have a resolving power near 23,000 and $\mathrm{S} / \mathrm{N}$ ratio of $65-75$ at $6700 \AA$. Properties of the stars are listed in Table 1 .

\begin{tabular}{cccccc}
\multicolumn{5}{c}{ Table 1. } & \multicolumn{4}{c}{ Observed stars in Terzan 7 } \\
\hline Star & V & V-I & $\begin{array}{c}\mathrm{T}_{\text {eff }} \\
(\mathrm{K})\end{array}$ & $\log \mathrm{g}$ & $\begin{array}{c}\xi_{\mathrm{t}} \\
\left(\mathrm{km} \mathrm{s}^{-1}\right)\end{array}$ \\
\hline S16 & 15.05 & 1.64 & $3985 \pm 50$ & $0.50 \pm 0.12$ & $1.9 \pm 0.1$ \\
S34 & 16.09 & 1.45 & $4255 \pm 60$ & $1.28 \pm 0.14$ & $1.6 \pm 0.1$ \\
S35 & 16.10 & 1.41 & $4280 \pm 50$ & $0.93 \pm 0.13$ & $1.8 \pm 0.1$ \\
\hline \hline
\end{tabular}




\section{Analysis}

We employed standard abundance analysis methods making use of Kurucz (1993) plane-parallel model atmospheres and the LTE abundance analysis code MOOG (developed and maintained by Chris Sneden). We have analyzed our spectra using both equivalent widths, and, for blended lines, spectrum synthesis. Atmospheric parameters were obtained as follows. For effective temperature, we selected the model from which we find no dependence of individual Fe I abundances on lower excitation potential. The microturbulence velocity parameter was determined by requiring that the individual $\mathrm{Fe} I$ line abundances show no correlation with equivalent width. The surface gravity was established by the usual requirement that $\mathrm{Fe}$ I and Fe II lines yield the same Fe abundance. Our derived abundances are listed in Table 2; the methods of analysis are also indicated: spectrum synthesis (SS) and equivalent width (EW).

Table 2. $\quad[\mathrm{X} / \mathrm{H}]$ values for Terzan 7 Stars

\begin{tabular}{lcccc}
\hline Element & S16 & S34 & S35 & mean \\
\hline OI,SS & $-0.36 \pm 0.08(1)$ & $-0.39 \pm 0.09(1)$ & $-0.35 \pm 0.08(1)$ & -0.36 \\
NaI,EW & $-0.86 \pm 0.12(2)$ & $-0.74 \pm 0.10(2)$ & $-1.38 \pm 0.10(1)$ & -0.92 \\
AlI,SS & $-0.66 \pm 0.10(2)$ & $-0.64 \pm 0.10(2)$ & $-0.70 \pm 0.15(1)$ & -0.66 \\
Si I,EW & $-0.39 \pm 0.16(2)$ & $-0.18 \pm 0.24(2)$ & $-0.45 \pm 0.12(4)$ & -0.37 \\
CaI,EW & $-0.49 \pm 0.20(2)$ & $-0.33 \pm 0.20(2)$ & $-0.36 \pm 0.09(2)$ & -0.39 \\
ScII,EW & $-0.48 \pm 0.13(1)$ & $-0.63 \pm 0.16(1)$ & $-0.89 \pm 0.15(1)$ & -0.67 \\
Sc II,EW & $-0.92 \pm 0.08(1)$ & $-0.64 \pm 0.11(1)$ & $-0.75 \pm 0.10(1)$ & -0.77 \\
Ti I,EW & $-0.41 \pm 0.10(2)$ & $-0.24 \pm 0.16(2)$ & $-0.47 \pm 0.11(2)$ & -0.37 \\
Fe,EW & $-0.68 \pm 0.04(26)$ & $-0.52 \pm 0.06(30)$ & $-0.62 \pm 0.05(34)$ & -0.61 \\
NiI,EW & $-0.79 \pm 0.08(2)$ & $-0.62 \pm 0.09(2)$ & $-0.89 \pm 0.10(2)$ & -0.77 \\
Ni I,SS & $-0.80 \pm 0.08(1)$ & $-0.40 \pm 0.09(1)$ & $-0.81 \pm 0.10(1)$ & -0.67 \\
Zr I,EW & $-0.90 \pm 0.12(2)$ & $-0.86 \pm 0.13(2)$ & $-1.00 \pm 0.14(2)$ & -0.92 \\
LaII,EW & $-0.22 \pm 0.09(1)$ & $-0.04 \pm 0.04(1)$ & $-0.45 \pm 0.10(1)$ & -0.24 \\
EuII,SS & $-0.13 \pm 0.08(1)$ & $+0.07 \pm 0.11(1)$ & $-0.14 \pm 0.10(1)$ & -0.07 \\
\hline \hline
\end{tabular}

\section{Discussion}

Our derived mean $[\mathrm{Fe} / \mathrm{H}]$ value for Terzan $7,-0.61 \pm 0.08 \mathrm{dex}$, is in good agreement with the photometric estimate by Sarajedini and Layden (1997) of $-0.82 \pm 0.15$ dex. The agreement with Sarajedini and Layden's result also supports their age estimate of 6 Gyrs younger than 47 Tuc.

\subsection{Comparison with 47 Tuc and M71}

1. The derived $[\mathrm{O} / \mathrm{Fe}]$ value of +0.25 is very similar to field stars and globular clusters in our galaxy with similar $[\mathrm{Fe} / \mathrm{H}]$ values. For example, Brown et al. (1990) found $[\mathrm{O} / \mathrm{Fe}]=+0.42 \pm 0.07$ dex in $47 \mathrm{Tuc}$, for which they found $[\mathrm{Fe} / \mathrm{H}]$ $=-0.9$ dex (though others have found $\approx-0.7$ dex and we find to be -0.62 
dex). For M71, whose $[\mathrm{Fe} / \mathrm{H}]=-0.79$ dex, Sneden et al. (1994) found $[\mathrm{O} / \mathrm{Fe}]$ $=+0.33 \pm 0.03$ dex for stars which do not show the high $\mathrm{Na}$, low $\mathrm{O}$ anomaly.

2 . For the $\alpha$-elements we have data for $\mathrm{Si}, \mathrm{Ca}$, and $\mathrm{Ti}$ and find a mean value for $[\alpha / \mathrm{Fe}]$ of +0.23 dex, which is slightly above that of field stars in our Galaxy, but falls within the scatter (McWilliam 1997). For M71 Sneden et al. (1994) found $[\alpha / \mathrm{Fe}]=+0.3 \mathrm{dex}$, while for 47 Tuc, Brown et al. (1990) found $[\alpha / \mathrm{Fe}]$ $=+0.28 \pm 0.10$ dex. Our Ca abundance for Terzan $7[\mathrm{Ca} / \mathrm{H}]=-0.39$, which is close to the metallicity derived from the CaII triplet of -0.36 (Da Costa and Armandroff 1995). We urge caution in deriving "metallicities" rather than $\mathrm{Ca}$ abundances from the Ca II triplet, especially for relatively metal-rich stars.

For comparison purposes, we also used the same methods to analyze spectra of two stars in 47 Tuc. The 47 Tuc stars are hotter and of lower luminosity than the Terzan 7 stars. We found $[\mathrm{Fe} / \mathrm{H}]=-0.62 \pm 0.10 \mathrm{dex}$, and $[\alpha / \mathrm{Fe}]=+0.34$ dex. For $[\mathrm{Eu} / \mathrm{Fe}]$ we found a value of +0.23 , a smaller excess than in Terzan 7 .

\section{2. $\mathrm{r} / \mathrm{s}$ process ratios}

Among the heavy elements in the Terzan 7 stars, $[\mathrm{Zr} / \mathrm{Fe}]=-0.31,[\mathrm{La} / \mathrm{Fe}]$ $=-0.37$, and $[\mathrm{Eu} / \mathrm{Fe}]=+0.54 \mathrm{dex}$. These ratios are consistent with an $\mathrm{r} / \mathrm{s}$ process ratio of $7.5 \pm 3$. This compares to $4 \pm 1$ for Arcturus (defined as 1 for the Sun); see Gonzalez and Wallerstein 1998 - Table 7.

\section{Conclusions}

1. Our mean metallicity of $[\mathrm{Fe} / \mathrm{H}]=-0.6$ dex for three giants in Terzan 7 agrees with the photometric value of Sarajedini and Layden and with that of disk globulars such as 47 Tuc and M71.

2. Our $[\mathrm{O} / \mathrm{Fe}]$ and $[\alpha / \mathrm{Fe}]$ value is similar to those in Galactic globulars of comparable $[\mathrm{Fe} / \mathrm{H}]$.

3. The $\mathrm{r} / \mathrm{s}$ process ratio for the stars in Terzan 7 falls above the ratio in the Sun and Arcturus.

4. It is clear that all captured globular clusters are not the same, which should not be a surprise to anyone.

\section{References}

Brown, J. A., Wallerstein, G., \& Oke, J. B. 1990, AJ, 100, 1561

Da Costa, G. S. \& Armandroff, T. E. 1995, AJ, 109, 2533

Gonzalez, G. \& Wallerstein, G. 1998, AJ, 116, 765

Kurucz, R. L. 1993, CD ROM 13, ATLAS9 Atmosphere Programs and 2 km/s Grid (Cambridge: Smithsonian Astrophys. Obs.)

Layden, A. C. \& Sarajedini, A. 2000, AJ, 119, 1760

McWilliam, A. 1997, ARA\&A, 35, 503

Sarajedini, A. \& Layden, A. 1997, AJ, 113, 264

Sneden, C., Kraft, R. P., Langer, G. E., Prosser, C. F. \& Shetrone, M. E. 1994, AJ, 107, 1773 